\title{
Lupus Nephritis in Qatar
}

N. Abdulla, O. Fituri, F. Alam, I. Mujeeb,

E. Satti, N. Hadwan, M. Hammoudeh

Hamad Medical Corporation, Medicine/Rheumatology, Doha-Qatar

Lesceagi:

Objectives- To review histological types, clinical manifestations, laboratory findings and treatment outcome for Lupus Nephritis (LN) cases in Qatar.

Design and Methods- We retrospectively analyzed the data of all patients with biopsy proven LN diagnosed between January 2006 and December 2014 at Hamad General Hospital, Doha-Qatar. Clinical, laboratory and treatment data were collected from electronic records. Outcome at the end of $1^{\text {st }}$ year of treatment was recorded.

Results- Our cohort included 50 patients; 43 (86\%) were females and $7(14 \%)$ were males with a ratio of $6: 1$.The median age at onset of $L N$ was 29 years. The average interval between onset of Lupus and diagnosis of Lupus nephritis was 2.5years.

The most common non-renal manifestations at the time of $L N$ diagnosis were arthralgia (50\%), arthritis (34\%), fever (34\%), and serositis (22\%).

Class 4 was the most common type (44\%), followed by class 3 (20\%), class 5 (12\%), class $2(12 \%)$, Class $3+5(6 \%)$, Class $2+5(4 \%)$ and class $1(2 \%)$ in our cohort.

Proteinuria alone was seen in $72 \%$ patients, renal insufficiency in $26 \%$ whereas nephrotic range proteinuria in $34 \%$ cases.

Anti-ds-DNA was positive in 90\% cases, Low C3 in $83 \%$ and low C4 in 59\% cases at the time of LN diagnosis.

Mycophenolate mofetil was the most commonly prescribed drug (74\%), followed by Azathioprine (14\%), and cyclophosphamide (6\%). Pulse steroid was prescribed in $44 \%$ of cases, and oral steroid in $100 \%$ cases.

Outcome at 1st year-end was available for 31 (62\%) cases, of which $38.7 \%$ achieved complete remission (CR), $25.8 \%$ partial remission (PR) and $35.5 \%$ had treatment failure (TF). CR was observed in $42.9 \%$ of class $4,42.9 \%$ of class 3 and $20 \%$ of class 5 LN patients.

Conclusion- The most common LN class in our cohort was Class 4, similar to those reported from most centers worldwide.

Further studies are required to look into the long term outcome of our patients, especially the Qatari and Arab patients. 